\title{
Etude de l'appareil radical de jeunes plants de Chênes verts dont le pivot se développe sans amputation, ni déviation
}

\author{
Y. BEISSALAH, T. AMIN, B. EL HAJZEIN et P. NEVILLE \\ avec la collaboration technique de Janine BERNARD \\ Laboratoire de Morphogenèse végétale, Faculté des Sciences et Techniques de St-Jérôme, \\ Université d'Aix-Marseille III, Bô̂te 442, Avenue Escadrille Normandie Niémen, \\ F 13397 Marseille Cedex $13^{(1)}$
}

\begin{abstract}
Résumé
L'appareil radical du jeune plant de Chêne vert (Quercus ilex L.) est constitué par une racine principale (pivot) à géotropisme positif, à croissance rapide et indéfinie portant de courtes racines latérales. Le pivot n'est pas rectiligne mais ondule aussi bien sur substrat meuble (tourbe ou terreau) que sans substrat (caisson à brouillard). Les plus grandes racines latérales naissent généralement sur la partie convexe d'une ondulation. Le pivot pousse mieux sur tourbe que sur terreau ou en caisson à brouillard. Il réagit rapidement à un abaissement de 25 à $20^{\circ} \mathrm{C}$ qui diminue sa vitesse de croissancce surtout sur terreau, moins sur tourbe et peu sur caisson à brouillard qui exerce un effet tampon. La vitesse de croissance du pivot montre des fluctuations indépendantes de la température ou du substrat (ou de son absence), dont la périodicité varie de deux à sept jours et qui sont vraisemblablement l'expression d'un rythme endogène irrégulier. Les racines latérales n'influent pas sur les différentes variations de croissance du pivot.

Le pivot a généralement 6 faisceaux ligneux majeurs (plus rarement 4 à 8) immédiatement sous les cotylédons et en relation avec ceux-ci ( 3 par cotylédon). Ces faisceaux se divisent entre le $3^{\mathrm{e}}$ et le $7^{\mathrm{c}}$ centimètre sous les cotylédons, ce qui peut aboutir au triplement du nombre de faisceaux ligneux. Ces faisceaux supplémentaires finissent par disparaître progressivement jusqu'au $20^{e} \mathrm{~cm}$ à partir des cotylédons. Au-delà, on retrouve le même nombre de faisceaux ligneux majeurs qu'au départ. Le nombre des faisceaux libériens, d'abord double de celui des faisceaux ligneux majeurs dans la région proche des cotylédons, diminue progressivement pour devenir égal à ce dernier au-delà du $20^{\mathrm{C}} \mathrm{cm}$ au-dessous des cotylédons. Les racines latérales ont entre 3 et 5 faisceaux ligneux.
\end{abstract}

Mots clés: Quercus ilex, racine, développement, plantule, température, faisceaux conducteurs.

\section{Introduction}

Le Chêne vert (Quercus ilex L.) est un constituant important de la forêt méditerranéenne, particulièrement en France. Une connaissance du déterminisme de l'architecture de l'appareil radical des jeunes plants issus de semis est d'un intérêt appréciable aussi bien dans un but d'applications pratiques qu'au plan fondamental.

(1) Travail réalisć avec laide de l'I.N.R.A. dans le cadre du G.R.E.C.O. Forêt Méditerranéenne (C.N.R.S.-I.N.R.A.). 
Bien que, depuis l'importante mise au point de Torrey (1965), un certain nombre de travaux concernant le développement des racines aient été réalisés (Feldmann, 1984 ; JACKSON, 1986), il n'existe pas, à notre connaissance, de travaux de ce type à propos du Chêne vert. Il nous a donc paru intéressant de contribuer à combler cette lacune en étudiant le développement de l'appareil radical du jeune plant. Avant de rechercher les corrélations entre organes qui contrôlent l'élaboration de son architecture et comment des obstacles peuvent modifier cette dernière, ce qui fera l'objet de publications ultérieures, nous présenterons ici la structure et le comportement, dans diverses situations, de l'appareil radical dont le pivot reste entier et non dévié.

\section{Matériel et méthodes}

\subsection{Matériel}

Notre étude concerne le développement de l'appareil radical de plants issus de semis chez Quercus ilex L. Ce taxon est compris dans ce travail au sens le plus large qui est celui des flores classiques (Costes, 1901 ; Fournier, 1946) et non au sens restreint de Schwartz (1964) dans "Flora Europaea".

Les semences utilisées sont des glands provenant d'arbres situés dans la région de Marseille. Ils sont conservés selon la méthode d'Aissa (1981). Pour atténuer l'hétérogénéité génétique, nous avons tenu à ce que, dans une expérience donnée, les différents lots soient constitués avec les semences provenant d'un même arbre.

\subsection{Méthodes}

Du semis à la fín des observations, les plantes se sont développées dans une pièce du laboratoire où la température varie de $11,5^{\circ} \mathrm{C}$ à $28^{\circ} \mathrm{C}$ et l'humidité relative de 22 à 98 p. 100 selon la période considérée. Pendant toute la durée des expériences on enregistre les variations de température et d'humidité dans cette pièce. La durée de l'éclairement, la qualité et la quantité de lumière varient selon la période des observations puisque les plantes reçoivent uniquement la lumière solaire par les fenêtres du laboratoire.

Le substrat généralement utilisé est la tourbe sauf indication contraire. Pour protéger nos plants des attaques, dues surtout aux champignons, l'eau de ville que nous utilisons pour l'humidification de cette tourbe est additionnée de cryptonol de manière à obtenir une solution à 0,020 p. 100 . C'est sur ce milieu que sont semés les glands débarrassés au préalable de leur péricarpe pour faciliter la germination (AIsSA, 1981). Cinq à six jours après la germination, lorsque les jeunes racines ont atteint entre 3 et $5 \mathrm{~cm}$, les semis sont transférés sur leur substrat définitif contenu dans des minirhizotrons (RIEDACKer, 1974). Ce sont des parallélépipèdes de $4 \times 30 \times 95 \mathrm{~cm}$ en matière plastique ayant une face amovible en PVC transparent. Ils sont inclinés à $30^{\circ}$ par rapport à la verticale, face transparente vers le bas.

Le contact forcé des racines contre la paroi transparente n'est probablement pas sans conséquence sur leur développement. Mais divers auteurs estiment que la croissance des racines observables à travers cette paroi est assez représentative de celle du système radical normal (TaYlor et al., 1970 ; HofFManN, 1974). 
Dans les minirhizotrons, les apports hydriques sont limités à la quantité d'eau contenue dans le substrat lors de son humidification initiale.

Dans certaines expériences, les semis sont transférés sur des rhizotrons à brouillard (LAMOND, 1975) où les racines peuvent pousser sans substrat, donc sans contrainte. Elles sont simplement humidifiées automatiquement à intervalles réguliers par une brumisation de solution nutritive. Dans ce système, on peut tenir compte de toutes les racines qui poussent.

Pour vérifier si la croissance du pivot est sensible à la présence des racines latérales, il a fallu supprimer ces dernières dans certains lots.

Les expériences sont conduites sur des lots de 12 plantes pour chaque condition. Il en survit de 7 à 12 selon les cas. Les minirhizotrons reçoivent 2 individus chacun, et sont disposés au hasard sur les portoirs, les différentes conditions étant mélangées. Un rhizotron à brouillard peut recevoir les deux lots cultivés dans ces conditions.

La croissance et l'orientation des racines sont notées à intervalles réguliers (de 1 à 3 jours selon les expériences) en décalquant chaque fois, sur des feuilles de plastique transparent, les différents accroissements, avec des marqueurs de couleurs différentes pour chaque date d'observation. La taille des racines est ensuite mesurée sur ces feuilles de plastique avec un curvimètre.

Les graphes traduisant les variations de la vitesse de croissance moyenne correspondent à de petits nombres de cas. Pour éliminer les fluctuations mineures, ces courbes ont été lissées par la méthode des moyennes mobiles $(\mathrm{Xi}=(\mathrm{xi}-1+\mathrm{xi}+\mathrm{xi}+1) / 3)$. Dans tous les cas, l'étude graphique des écarts $e_{i}=X_{i}-x_{i}$ nous a permis de constater qu'il n'existe pas de variations rythmiques régulières dont la période soit inférieure à 3 jours.

Pour rechercher si la vitesse de croissance du pivot est indépendante ou liée aux variations de température, le coefficient de corrélation entre ces deux paramètres a été calculé pour les différentes situations (substrats divers ou absence de substrat).

Sauf pour quelques contrôles et examens préliminaires réalisés sur des coupes faites à main levée, l'essentiel de l'étude histologique porte sur des coupes sériées de matériel fixé au CrAF III, inclus dans la paraffine et colorées à la safranine, à l'hématoxyline et au bleu d'aniline. Les coupes, généralement transversales, ont une dizaine de micromètres d'épaisseur.

\section{Résultats et discussion}

Nous décrirons l'élaboration de l'appareil radical de jeunes plants entiers, puis les différences anatomiques et leurs variations pour les différents types de racines.

\subsection{Elaboration de l'appareil radical de jeunes plants}

L'appareil radical du jeune plant est essentiellement formé par une racine principale ou pivot portant des racines latérales ou secondaires (fig. 1). Cette architecture est classique chez de nombreux arbres et a été observée chez tous les chênes précédemment étudiés qu'ils soient caducifoliés, Q. robur L. (ChAmpagnat et al., 1974 ; RiE- 


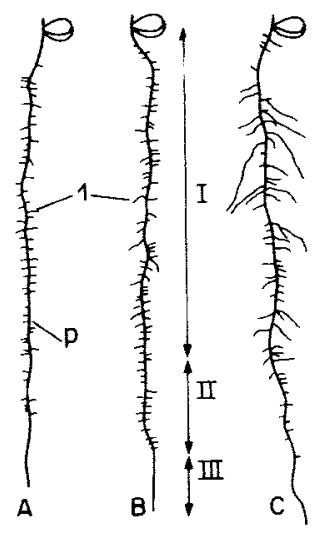

FIG. 1 .

Schéma de lappareil radical de 3 plants de Chêne vert développés sur tourbe et dont le pivot (p) mesure une cinquantaine de centimètres. Les 3 individus $A, B$ et $C$ se distinguent par la densité et la taille moyenne des racines latérales. En $A$ et $C$ la densité est à peu près la même (73 racines latérales apparentes pour $A$ et 77 pour $C)$ et relativement faible, alors qu'elle est forte pour $B$ (122 racines latérales).

Les racines latérales restent courtes pour $A$ et $B$, mais sont souvent nettement plus allongées pour $C$. Les étages I, II et III sont caractérisés par des racines latérales dans des états différents.

Dans l'élage I, les racines latérales ont terminé leur croissance;

en II. elles sont en cours de croissance et en III elles ne sont pas encore apparues.

Diagram of the root system of 3 Holly Oak (Quercus ilex) grown on peat and with a tap root about 50 centimeter long. The 3 individuals $A, B$ and $C$ can be distinguished by the density and mean length of their branch roots. For $A$ and $C$, density is similar (73 visible branch roots for $A$ and 77 for $C)$ and relatively low compared to the high density of $B$ (122 branch roots).

Branch roots remain short for $A$ and $B$ but are often distinctly longer for $C$.

Levels I, II and III are characterized by branch roots in different conditions.

In level I branch roots finished their growth. In level II they are undergoing growth and in level III they are not visible yet.

DACKer, 1976a ; TAVAKol, 1979), $Q$. petraea et $Q$. pubescens (observations non publiées), ou sclérophylles, Q. suber L. (Riedacker, 1976a et b ; El Alaoui, 1978) et Q. coccifera (observation non publiée).

Il arrive que les racines latérales portent des racines tertiaires, mais ces dernières ne se développent véritablement que sur des racines latérales ayant subi une stimulation, racines que nous n'étudierons pas ici.

Nous distinguerons donc, pour décrire macroscopiquement le système juvénile, le pivot puis les racines latérales.

\subsection{Le pivot}

Le pivot résulte de l'allongement de la radicule. Il croît verticalement vers le bas. $\mathrm{Si}$ un obstacle le détourne temporairement de cette direction de croissance, il la retrouve dès que l'obstacle est contourné. Ce comportement est l'expression d'un géotropisme positif. 
Un examen attentif montre que, en réalité, le pivot ne pousse pas de manière parfaitement rectiligne. Il ondule en général faiblement autour de la direction verticale. D'autres auteurs, ayant noté que ces ondulations sont accentuées sur substrat peu pénétrable, pensent qu'elles résultent de la résistance du substrat (RIEDACKer, 1976a ; TolHen, 1977). Pourtant, chez le Chêne vert, le pivot continue à onduler de manière nette dans les caissons à brouillard où il n'est soumis à aucune contrainte mécanique. Les ondulations constatées seraient donc dues à une autre cause : interférence de divers gradients du milieu avec la gravité ou interactions de croissance avec les racines latérales? Il est difficile de trancher, mais on peut souligner que la suppression régulière des racines latérales au fur et à mesure qu'elles apparaissent n'empêche pas les ondulations du pivot. Ces ondulations pourraient aussi être l'expression d'une rythmicité endogène de la croissance qui semble exister chez le Chêne vert comme nous le verrons plus loin.

Dans nos conditions expérimentales, la vitesse de croissance pour la durée des observations est de $1,7 \mathrm{~cm} \cdot \mathrm{j}^{-1}$ (écart-type 0,2 ou 0,3 , pour 5 répétitions, sur tourbe), de $1,4 \mathrm{~cm} \cdot \mathrm{j}^{-1}$ (écart-type 0,4 , pour 2 répétitions, sur terreau) et $\mathrm{de} 1,4 \mathrm{~cm} \cdot \mathrm{j}^{-1}$ en caisson à brouillard (écart-type 0,2 , pour 2 répétitions), ce qui est nettement supérieur à celle observée chez $Q$. suber : $0,7 \mathrm{~cm} \cdot \mathrm{j}^{-1}$ (EL Alaoul, 1978). Toutefois, cette vitesse n'est pas constante, elle tend à diminuer après 1,5 à 2 mois, parfois jusqu'à l'arrêt complet.

A partir de ce stade, une même racine principale peut ensuite présenter 2 ou 3 arrêts de 2 à 6 jours entrecoupés de 2 à 10 jours de croissance.

Pour essayer de préciser les causes de ces fluctuations de croissance les expériences suivantes ont été réalisées.

Divers lots de plantes sont constitués pour suivre la croissance du pivot dans différentes conditions. Les cultures ont lieu dans une même salle, soit sur minirhizotrons de type RIEDACKER, soit sur rhizotrons à brouillard de type LAMOND. Dans les minirhizotrons, le substrat est constitué soit par de la tourbe (série des lots A), soit par du terreau (série des lots B) mouillés avec de l'eau de ville. Dans les rhizotrons à brouillard, la culture a lieu sans substrat (série des lots C). L'alimentation des plantes est ici assurée par un brouillard d'eau de ville dans laquelle on a fait tremper de la tourbe pendant 3 jours et que l'on a récupérée par filtration.

Les cultures sont mises en route le même jour à raison de deux lots dans chaque condition. Dans les lots $\mathrm{A}, \mathrm{B}$ et $\mathrm{C}$, les pivots conservent leurs racines latérales alors qu'elles sont supprimées au fur et à mesure qu'elles apparaissent dans les lots $\mathrm{A}^{-}, \mathrm{B}^{-}$et $\mathrm{C}^{-}$. Dans la série $\mathrm{A}$, sur tourbe, on place en outre des lots qui commencent à se développer 2 (A2), 4 (A4) ou 6 (A6) jours après la mise en route des lots initiaux.

Les accroissements des pivots sont notés tous les jours au même moment de la journée.

Pour ne faire apparaître d'abord que les fluctuations majeures, on étudie les courbes lissées par la méthode des moyennes mobiles (fig. 2). On constate alors que, dans les trois séries $A, B$, et $C$, ces fluctuations majeures présentent une nette coïncidence pour les six lots $\mathrm{A}, \mathrm{A}^{-}, \mathrm{B}, \mathrm{B}^{-}$et $\mathrm{C}, \mathrm{C}^{-}$. Si l'on examine dans le même temps les variations quotidiennes des températures moyennes (moyenne entre les températures maximale et minimale pour chaque jour), on constate que les fluctuations majeures de la courbe obtenue après lissage (fig. 3, I) coïncident avec celles des accroissements quotidiens des pivots (fig. 2). 


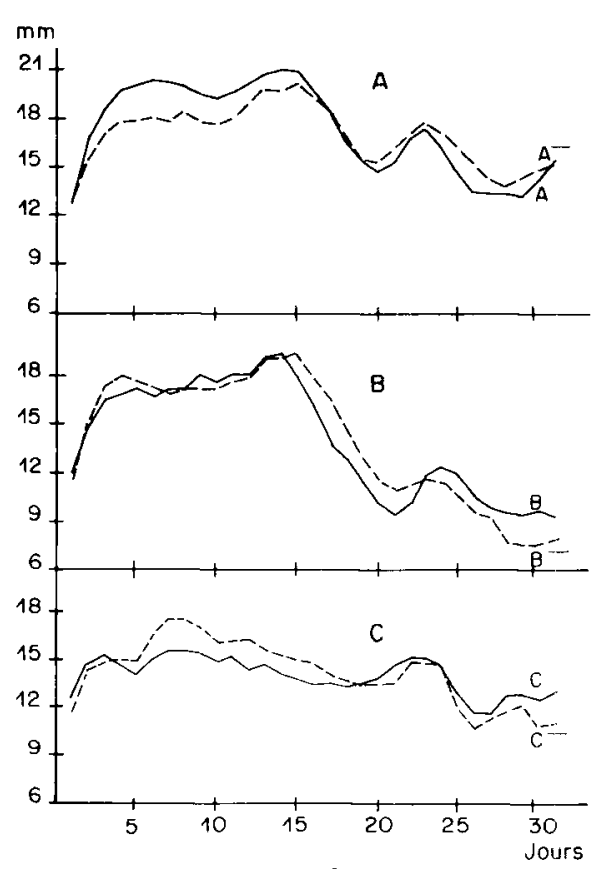

Fig. 2

Variation de l'accroissement moyen quotidien du pivot poussant sur tourbe (A), sur terreau (B) ou en caisson à brouillard (C). Les lots $A, B$ et $C$ possèdent leurs racines latérales alors que les lots $A^{-}, B^{-}$et $C^{-}$en sont régulièrement privés.

Chaque point correspond à la valeur moyenne pour 7 à 12 individus selon les lots, après lissage par la méthode de la moyenne mobile.

Variation of tap root mean daily increment : $A$ on peat, $B$ on loam and $C$ in fog chamber.

Groups $A, B$ and $C$ have branch roots while those of groups $A^{-}, B^{-}$and $C^{-}$ are regularly removed.

Each point corresponds to the mean value of 7 to 12 individuals, depending on the group. Smoothing of the curve was obtained by the moving average method.

Le calcul des coefficients de corrélation entre la température d'une part et les vitesses de croissance du pivot dans les diverses situations d'autre part montre une corrélation généralement très significative (pour un degré de liberté de $28, r=0.8036$ pour $\mathrm{A} ; 0,7941$ pour $\mathrm{A} ; 0,8394$ pour $\mathrm{B} ; 0,7962$ pour $\mathrm{B}^{*} ; 0,5842$ pour $\mathrm{C}$ signification à mieux que 1 p. 100 dans tous ces cas $; 0.3441$ pour $\mathrm{C}^{-}$signification seulement à mieux que 7 p. 100).

La croissance du pivot est donc fonction de la température. Ce sont les plantes cultivées sur terreau qui sont les plus sensibles (fig. 2, B), puis celles sur tourbe (A) et enfin celles sans substrat $(C)$, l'ambiance du rhizotron à brouillard semblant avoir un effet tampon.

Si l'on observe les graphes obtenus après lissage pour les lots placés sur tourbe à 0 (A), 2 (A2), 4 (A4) ou 6 (A6) jours (fig 3, II), on constate bien que les fluctuations majeures sont en relation avec les variations des températures moyennes quotidiennes et ne correspondent pas à un éventuel rythme de croissance de la plante, indépendant 


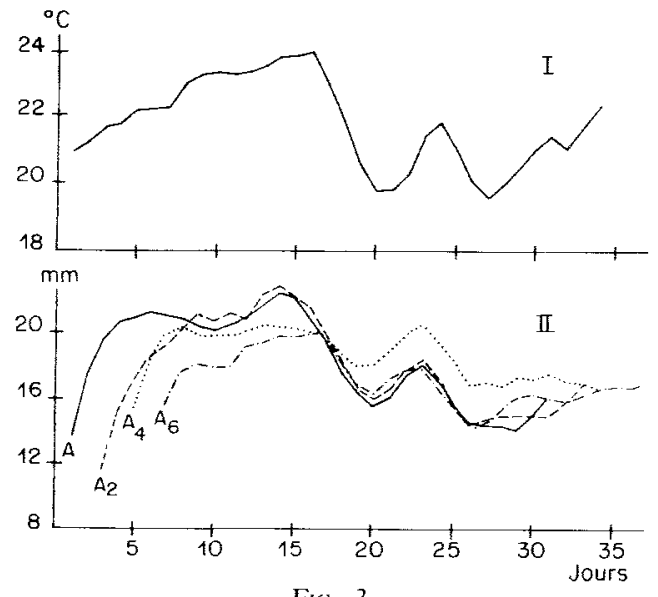

Fici. 3

Comparaison entre les variations de la température moyenne quotidienne (I) et les variations de l'accroissement moyen quotidien du pivot (II) cultivé sur tourbe à partir de 0 jour $(A)$ ou après 2,4 ou 6 jours du fait de germinations différées d'autant (A2, A4 et A6). Toutes les courbes (I et II) sont lissées par la méthode de la moyenne mobile. Chaque point du graphe II correspond à la moyenne pour 7 à 12 individus selon les lots.

Comparison between variations of the mean daily temperature (I) and variations of the tap root's mean daily increment (II) on peat, starting at day $O$ (A) or after 2, 4 or 6 days due to differed germinations (A2, A4 and A6).

Both curves were smoothed by the moving average method.

Each point in the diagram II corresponds to the mean value of 7 to 12 individuals, depending on the group.

des conditions du milieu (coefficient de corrélation $r=0,8358$ pour $A_{2} ; 0,7942$ pour $A_{4}$ et 0,4318 pour $A_{6}$ avec un d.d.l. respectivement de 26,24 et 22. Corrélation significative à mieux que 1 p. 100 pour $A_{2}$ et $A_{4}$, à mieux que 5 p. 100 pour $A_{6}$ ). Mais, on peut noter que l'incidence des fluctuations thermiques dépend de l'âge du pivot. Plus l'abaissement thermique intervient tôt, moins son influence est importante (Lots $A_{\downarrow}$ et surtout $A_{6}$ ), peut-être, en partie, parce que la vitesse de croissance n'a pas eu le temps d'atteindre des valeurs aussi importantes que pour les pivots plus âgés (lot $\mathrm{A}$ et $\mathrm{A}_{2}$ ).

Les mentions de l'influence de la température sur la croissance des racines sont nombreuses dans la littérature, mais elles concernent le plus souvent des végétaux herbacés (COOPER, 1973) ou des résineux (BARney, 1951; Arbez, 1971; Riedacker, 1976c ; Tolhen, 1977), plus rarement des feuillus comme Robinia pseudoacacia (SEELGER, 1956 ; HofFmanN, 1968). D'une manière générale, jusqu'à une valeur optimale, l'élévation de la température du sol favorise la croissance des racines. Au-delà, elle exerce un effet de plus en plus défavorable. Si une telle situation existe chez le Chêne vert, les conditions thermiques testées comprises entre 19.5 et $24,5^{\circ} \mathrm{C}$ sont situées endeçà de l'optimum. La réponse des racines aux fluctuations thermiques est rapide, ce qui a déjà été signalé pour Cedrus (TolHes, 1977).

Chez Quercus ilex, le pivot pousse plus vite sur tourbe (fig. 2, A), voire sur terreau (B) que dans des caissons à brouillard (C). Pourtant, habituellement, on observe que la croissance des racines est d'autant plus faible que le sol est plus résistant (Levy, 1968). Chez Cedrus atlantica, dans un milieu relativement peu pénétrable le 
pivot s'allonge moins vite, mais il porte des ramifications plus longues (RIEDAcKer, 1976a ; TOIHEN, 1977). La croissance moins rapide sur substrat peu pénétrable peut être due à une plus forte production d'acide abscissique (TIETz, 1975). Ces différentes données ne sont donc pas en accord avec nos résultats sur caisson à brouillard où la résistance est nulle. Il est possible qu'une faible résistance, entraînant une production plus limitée d'acide abscissique, favorise l'allongement du pivot comme le suggère le fait que de faibles concentrations de cet inhibiteur stimulent l'élongation de la racine de Pisum (GaITHer et al., 1975). Mais on ne peut écarter l'hypothèse que les acides organiques excrétés par les racines libèrent des éléments minéraux contenus dans les substrats (tourbe ou terreau), ces éléments favorisant la croissance du végétal (Com. verbale J.S. Frossard).
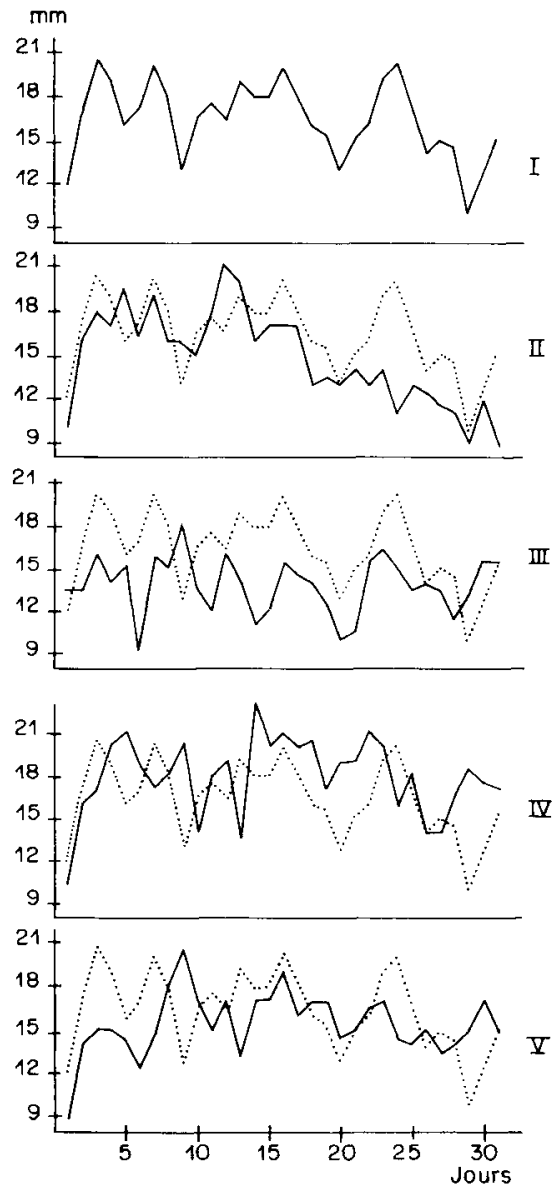

Fig. 4

Variations de l'accroissement quotidien du pivot de cinq individus (I à $V)$ du lot $A$ cultivé sur tourbe (voir fig. 2). Pour pouvoir comparer plus facilement les cinq individus entre eux, le graphe correspondant à l'individu I a été répété en pointillé dans les graphes II à $V$.

Variations of tap root daily increment for 5 group $A$ individuals (I to V) grown on peat (cf. fig. 2).

For an easier comparison of the 5 individuals, the graph related to individual $I$ was redrawn in doted-line in graphs II to $V$. 
Pour essayer de préciser la signification des fluctuations mineures observées sur les courbes non lissées d'allongements quotidiens moyens des pivots, les courbes individuelles non lissées ont été tracées (fig. 4).

On constate tout d'abord que ces fluctuations existent même dans les cultures sans substrat (série C) ce qui exclut de les attribuer à des variations de la résistance du substrat à la pénétration du pivot. Si l'on exclut les fluctuations de 1 ou $2 \mathrm{~mm}$ d'amplitude qui sont dans la marge d'erreur des mesures, il reste celles dont l'amplitude varie de 2 à $23 \mathrm{~mm}$ et qui sont parfois les seules observées sur certains graphes. La croissance du pivot se produit donc bien avec un rythme irrégulier dont la période varie de 2 à 7 jours. Comme les fluctuations des différents graphes correspondant aux pivots d'un même lot ne coüncident pas, il ne semble pas possible de les interpréter comme des conséquences de fluctuations d'un facteur du milieu. De plus, l'absence des racines latérales ne semble pas avoir de conséquences nettes (fig. 2).

Il apparaît donc que les pivots de Chêne vert ont une croissance qui se produit avec un rythme endogène irrégulier.

De nombreuses observations indiquent que l'activité de l'appareil radical est périodique, soit synchrone de l'activité de l'appareil aérien, soit le plus souvent, alternant avec celle-ci (Arbez, 1971 ; Souidi et Gagnaire-Michard, 1974 ; Riedacker, 1976c). L'apparition de nouvelles racines semble aussi être un phénomène rythmique se produisant toutes les trois à cinq semaines chez la plupart des végétaux ligneux (RIEDACKER, 1978). La vitesse moyenne d'allongement des racines est plus importante la nuit que le jour chez de nombreux végétaux ligneux (HeAd, 1965 ; Lyr et HoffmanN, 1967; Hilton et Khatamian, 1974) y compris chez Quercus robur (Kazarjan et Khurshudjan, 1965). La croissance de la racine principale ralentit chaque fois qu'elle forme des racines latérales (Priestley et Evershed, 1922 ; Priestley et Pearsall, 1922). Si toutes les variations de croissance dont il vient d'être question sont bien rythmiques, elles semblent, dans tout les cas, résulter soit d'influences climatiques, soit d'influences corrélatives d'autres parties de la plante. Pourtant, Arbez (1971) indique que l'activité radicale de Pinus nigra ssp. laricio obéit à une périodicité interne susceptible d'être modifiée par le jeu des facteurs du milieu. Mais l'existence de rythmes de croissance de périodicité interne chez les racines paraît peu probable à Kramer et Koslowski (1960) du fait qu'elles n'ont pas de période de dormance propre! Nos résultats plaident pourtant en faveur de l'existence d'un tel rythme qui n'implique pas d'arrêt total temporaire de la croissance, mais de simples ralentissements et accélérations de celle-ci.

\subsection{Les racines latérales}

Le pivot de 2 semaines mesure de 8 à $12 \mathrm{~cm}$. Il forme alors des racines latérales dans une zone s'étalant sur 2 ou $3 \mathrm{~cm}$ sous les ctyolédons. Bien qu'en nombre et à distance les unes des autres variables, elles apparaissent pour l'essentiel en ordre acropète (c'est-à-dire la première vers la base et les autres successivement de plus en plus loin de celle-ci). Leur apparition a lieu à une distance moyenne de $7 \mathrm{~cm}$ du sommet du pivot. Ces racines latérales sub-horizontales s'allongent peu, comparativement au pivot (fig. 1, A et B). A maturité, leur longueur moyenne est de $8 \mathrm{~mm}$. La figure 5 montre l'histogramme de la fréquence des longueurs de racines latérales insérées sur des pivots âgés de plus de 75 jours. Leur durée de croissance n'excède généralement pas une semaine, cependant, sur un pivot qui porte plus de 200 racines latérales, il arrive fréquemment qu'on en dénombre 1 à 3 p. 100 qui mesurent de 5 à 
$30 \mathrm{~cm}$ à la suite d'une croissance qui aura duré plus d'une semaine (fig. 5). Ces dernières sont alors plutôt obliques ou courbes (fig. 1, C) qu'horizontales et peuvent croître sur le mode indéfini. Elles apparaissent pour la plupart, sur la face convexe des faibles courbures du pivot, correspondant à son cheminement sinueux.

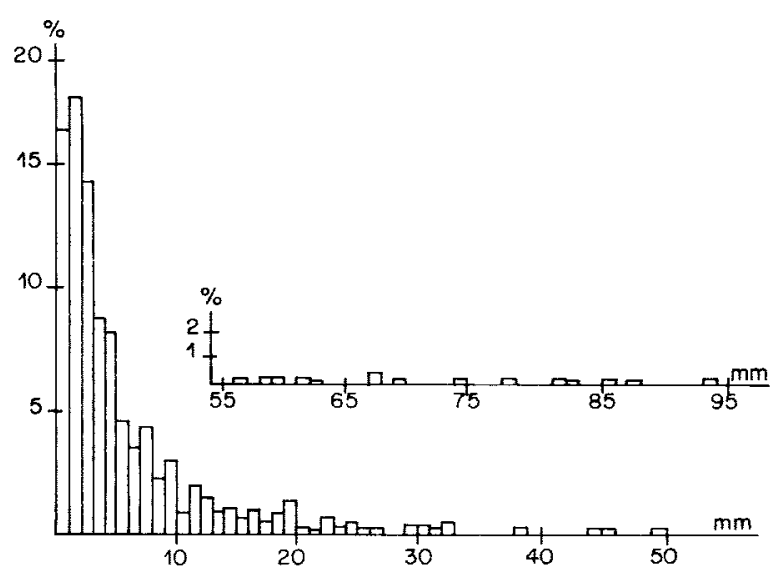

FIG. 5

Fréquences des racines latérales (ordonnée) de pivots témoins

en fonction de leur longueur finale en $\mathrm{mm}$ (abscisse). Le graphique concerne 4 individus.

Branch root frequency (vertical axis) on reference tap roots in relation to their final lenght in millimeters (horizontal axis). The graph was made with 4 individuals.

Si la courbure est prononcée, ce qui témoigne vraisemblablement d'une gêne temporaire importante dans la croissance du pivot, la racine latérale apparue sur la face convexe peut se redresser complètement.

Ces racines latérales à développement accru peuvent aussi se former après l'arrêt de croissance du pivot, soit lors de sa reprise, soit du fait de son arrêt définitif. Ceci suggère l'existence de corrélations de croissance du type dominance apicale que nous préciserons ailleurs.

Du fait de leur ordre d'apparition acropète et de leur croissance ordinairement limitée, les racines latérales d'un pivot à croissance rapide permettent de distinguer sur ce dernier trois parties (fig. 1).

L'étage I porte les racines latérales ayant terminé leur élongation. Chez un pivot témoin mesurant $50 \mathrm{~cm}$, il s'étend des cotylédons jusqu'à $32,6 \mathrm{~cm}$ en moyenne de ceuxci (cette longueur varie de 23 à $40 \mathrm{~cm}$ ).

L'étage II porte des racines jeunes, en croissance active, sa longueur varie de 6 à $20 \mathrm{~cm}$ avec une moyenne de $10,2 \mathrm{~cm}$.

Quant à l'étage III, il est constitué par la zone du pivot comprise entre le front d'apparition des racines latérales et son sommet. Cette zone est hétérogène puisqu'elle englobe le territoire proximal où des racines latérales, bien que non visibles extérieure- 
ment, sont en cours d'initiation, et le territoire distal où la formation des racines latérales n'a pas commencé et qui correspond à la zone méristématique et d'élongation du pivot.

Cette division du pivot en 3 étages, bien que grossière, nous permettra d'étudier ailleurs le comportement des racines latérales en fonction de leur stade de développement.

Lorsqu'elle atteint environ $3 \mathrm{~cm}$ de longueur, la racine latérale peut porter à son tour des racines tertiaires. Ces dernières mesurent moins d'un millimètre sur une racine latérale non stimulée.

\subsection{Etude anatomique}

Nous examinerons tout d'abord les modifications histologiques intervenant depuis la mise en place des cellules méristématiques jusqu'à l'apparition des racines latérales, puis nous étudierons les variations du nombre de faisceaux ligneux primaires dans les divers types de racines.

\subsection{Etapes de la maturation histologiques du pivot}

Une étude rapide montre que, dans l'étage III, dans une zone située à moins de $2 \mathrm{~cm}$ de l'apex du pivot, on peut déjà reconnaître les tissus conducteur et l'endoderme.

A $4 \mathrm{~cm}$ du sommet, l'assise péricyclique, située immédiatement sous l'endoderme, montre des divisions périclines correspondant à la différenciation de l'assise génératrice subéro-phellodermique. Le cambium libéro-ligneux s'individualise un peu plus tard.

Les premières ébauches de racines latérales sont contemporaines des premiers cloisonnements cambiaux. Elles se forment systématiquement à partir du péricycle et en face d'un pôle de bois primaire.

L'élimination du cortex est un phénomène qui fait suite à la croissance en épaisseur de la racine due à l'activité du cambium libéro-ligneux et de l'assise subérophellodermique. Leur fonctionnement détermine une pression radiale au niveau du cortex dont les cellules s'écrasent d'abord dans une progression centrifuge. Ensuite, il éclate en différents endroits laissant, pendant un temps, des lambeaux accrochés au suber.

\subsection{L'appareil conducteur primaire}

A partir de la germination (marquée par l'apparition de la radicule), il s'écoule plus de 10 jours avant que l'extrémité de l'épicotyle apparaisse. Pendant cette période, le pivot, qui s'accroît rapidement, ne peut être alimenté que par les cotylédons. Cela suggère que les appareils conducteurs de ces deux organes doivent être en relation directe. Pour vérifier cette hypothèse, nous avons effectué des coupes transversales sériées, depuis les pétioles cotylédonaires jusqu'à 2 ou $3 \mathrm{~mm}$ au-dessous de l'insertion des cotylédons, sur des individus âgés de 1 à 3 semaines.

La section transversale du pétiole cotylédonaire (fig. 6, A) montre 4 faisceaux libéro-ligneux dont deux très proches sont sub-médians, et les deux autres latéraux.

Lors de la fusion des bases cotylédonaires avec l'épicotyle, on voit que l'anneau procambial de ce dernier (b) s'élargit (c). Il s'ouvre de six brêches dans lesquelles 


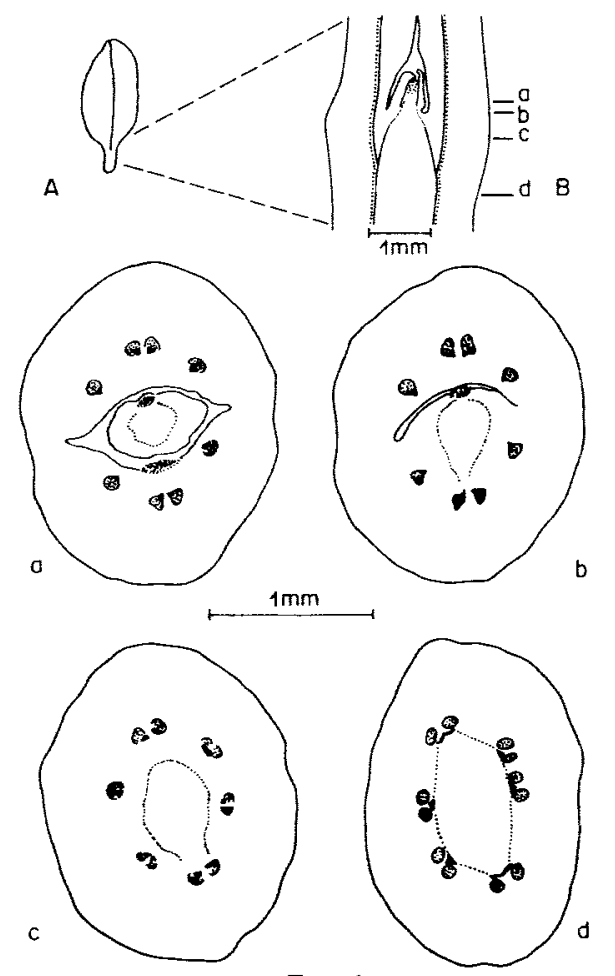

FiG. 6

Evolution de lappareil vasculaire depuis la base des cotylédons jusqu'à la radicule. A. - Vue générale de l'embryon. B. - Schéma d'une coupe longitudinale de l'embryon dans la région de l'insertion des cotylédons montrant les divers niveaux où ont été réalisées les coupes transversales a à d.

Vascular system evolution from the bottom of the cotyledons to the radicle.

A. - General view of the embryo. B. - Diagram of the embryo's longitudinal section in the cotyledons area, showing the different levels where cross-sections a to $d$ were made.

viennent s'insérer d'abord les couples de faisceaux sub-médians, puis les faisceaux latéraux (d). Le diamètre du cylindre central du pivot diminue régulièrement au fur et à mesure qu'on s'éloigne de ce niveau.

A ce stade, l'anneau de tissu vasculaire et provasculaire est constitué d'éléments en relation soit, avec les cotylédons pour les huits faisceaux libéro-ligneux, soit, avec l'épicotyle pour les parties de l'anneau intercalées entre ces six faisceaux ou couples de faisceaux. La partie en relation avec l'épicotyle peut contenir de petits faisceaux ligneux, à raison de un ou deux, entre les faisceaux d'origine cotylédonaire. Généralement, ces faisceaux disparaissent à 1 ou $2 \mathrm{~cm}$ au-dessous de l'insertion des cotylédons et vont exceptionnellement au-delà de $5 \mathrm{~cm}$.

Quant aux faisceaux issus des cotylédons, ils subissent, dans le centimètre sousjacent, une série de transformations correspondant au passage de la structure de la tige à celle de la racine, phénomènes bien étudiés chez de nombreuses espèces (TRONCHET, 1930 ; Duchaigne, 1951; Boureau, 1952; Lescot, 1967). Indiquons simplement 
(fig. 6, B) que les faisceaux latéraux subissent un élargissement, puis un dédoublement (c) et qu'ensuite les deux moitiés ligneuses se ressoudent en même temps que le pôle devient exarche, intercalé entre les deux masses libériennes (d). Parallèlement, la partie ligneuse des deux paires de faisceaux médians s’étirent aussi tangentiellement, puis se soude, dans chaque paire, au niveau du pôle qui devient unique et exarche intercalé entre les deux massifs libériens initiaux de chaque paire (d).

A $1 \mathrm{~cm}$ sous les cotylédons, la structure typique comprend 6 faisceaux ligneux majeurs alternant avec 6 paires de faisceaux libériens majeurs. On peut en outre observer, intercalés entre ceux-ci, les petits faisceaux en relation avec l'épicotyle (fig. 7 , $A^{\prime}$, s) qui vont disparaître un peu plus bas.

Entre 3 et $7 \mathrm{~cm}$ sous les cotylédons, les faisceaux ligneux majeurs tendent à s'élargir en deux ailes qui s'étirent tangentiellement et finissent par se séparer de la partie centrale (fig. $7, \mathrm{~B}$ ). Ce phénomène peut être unilatéral (fig. $7, \mathrm{~B}^{\prime}, \mathrm{f} 4$ et f5) ou même absent pour certains faisceaux $\left(B^{\prime}, f 3\right)$. Il en résulte la mise en place de faisceaux supplémentaires (maximum théorique 12, maximum observé 10) qui paraissent au départ dépourvus de pôle (fig. 7, B). Plus bas, entre 5 et $20 \mathrm{~cm}$, ce nombre diminue soit par fusion, soit par disparition progressive. Parallèlement les faisceaux ligneux supplémentaires qui persistent tendent à être organisés normalement avec un pôle ligneux exarche typique (fig. 7, C).

Le nombre total de faisceaux ligneux qui est maximal entre 5 et $7 \mathrm{~cm}$ (de 10 à 15) n'est plus que de 5 à 8 à $15 \mathrm{~cm}$ des cotylédons et se stabilise entre 20 et $25 \mathrm{~cm}$ où il revient au nombre des faisceaux majeurs (fig. 7, D), observé à $1 \mathrm{~cm}$ sous les cotylédons. Donc, seuls persistent les faisceaux ligneux majeurs. Dans le même temps, entre le $5^{\mathrm{e}}$ et le $25^{\mathrm{e}}$ centimètre, les faisceaux libériens majeurs tendent à fusionner deux à deux dans les intervalles entre les faisceaux ligneux majeurs (fig. 7, $\mathrm{B}^{\prime}, 1$ ) et leur nombre est régulièrement le même que celui des faisceaux ligneux au $25^{\circ}$ centimètre. Ensuite, sur plus de $30 \mathrm{~cm}$, le plan d'organisation de la vascularisation primaire reste stable.

Le nombre de faisceaux ligneux des racines latérales, quelle que soit l'importance de leur développement, varie entre 3 et 5 .

Le diamètre du cylindre passant par les pôles ligneux (non affecté par la croissance en épaisseur) est de $460 \mu \mathrm{m}$ en moyenne au milieu d'un pivot de $54 \mathrm{~cm}(690 \mu \mathrm{m}$ à sa base et $320 \mu \mathrm{m}$ près de l'apex) alors qu'il est de $150 \mu \mathrm{m}$ pour une racine latérale courte ou de $200 \mu \mathrm{m}$ au milieu d'une racine latérale atteignant $38 \mathrm{~cm}$.

Il est classique que les racines latérales aient un nombre de faisceaux ligneux inférieur à celui du pivot (Dyanat-Nejad et Neville, 1973 ; Riedacker, 1976a). Chez Quercus suber le pivot présente 13 à 14 faisceaux et la racine latérale 2 à 5 (EL AlaOUi, 1978).

Le phénomène d'augmentation puis de diminution du nombre de faisceaux ligneux parallèlement à une diminution régulière du diamètre du cylindre central rappelle ce que Dyanat-Nejad et Neville (1973) ont décrit à propos du pivot du jeune plant de Cacaoyer. Ils ont montré que la variation du nombre de faisceaux résulte d'une influence cotylédonaire et qu'elle ne peut s'exprimer si l'on empêche cette dernière de s'exercer. Par conséquent, seul le nombre de faisceaux ligneux majeurs doit être considéré comme une caractéristique propre du pivot. Ceci amène à s'interroger sur la signification des 13 ou 14 faisceaux indiqué par $E_{L}$ Alaour (1978) pour le pivot de Quercus suber. 

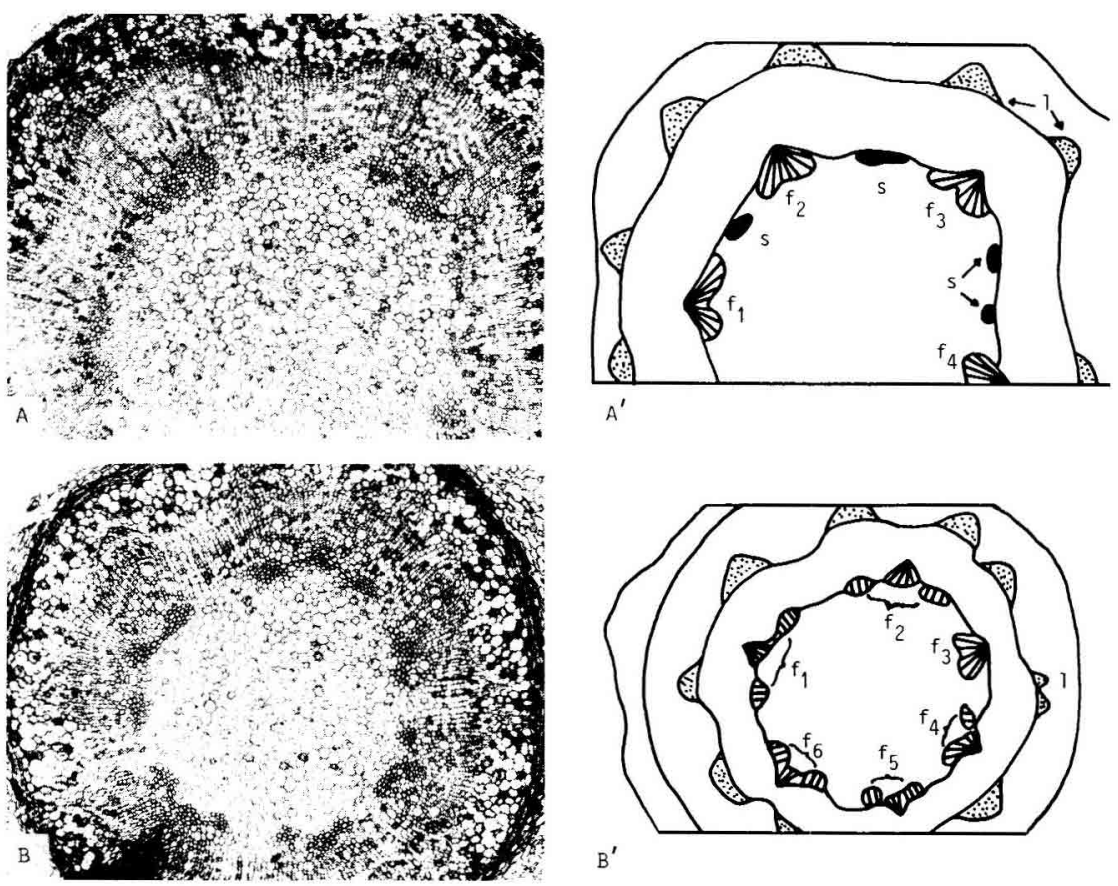

$\mathrm{B}^{\prime}$
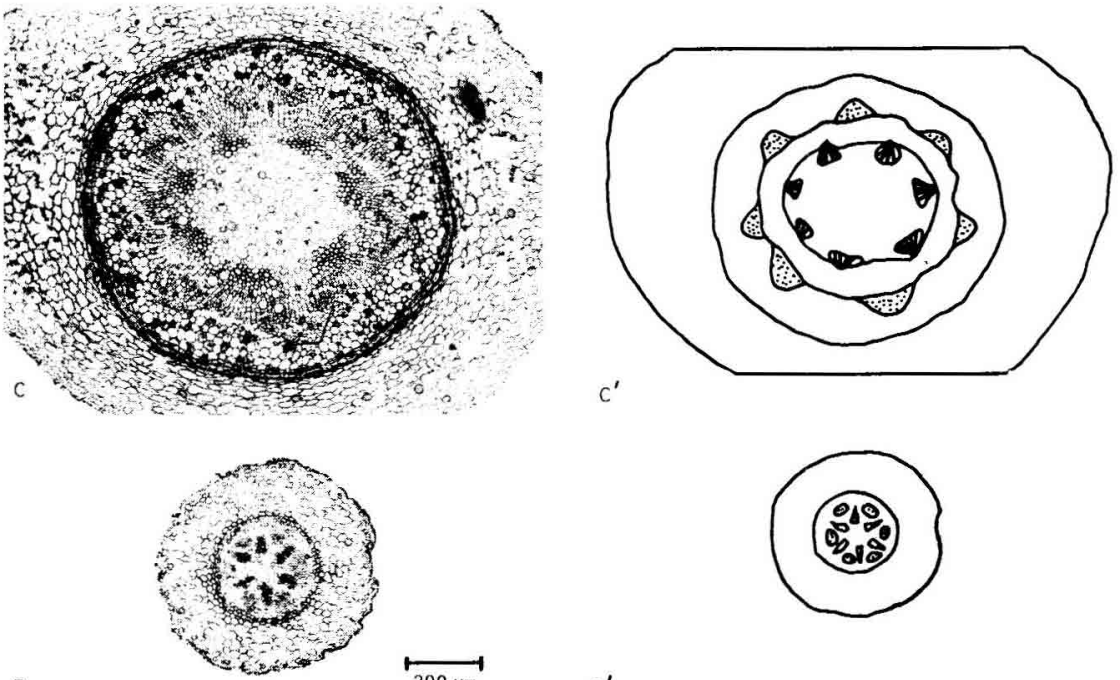

D

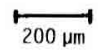

FIG. 7

Coupes transversales du pivot à $5(A), 7(B), 20(C)$ et $55 \mathrm{~cm}$ (D) des cotylédons et schémas á interprétation corresponáants $\left(A^{\prime}\right.$ à $\left.D^{\prime}\right)$.

$A-A^{\prime}:$ On distingue 4 faisceaux ligneux majeurs (fl à f4)

entre lesquels sont intercalés des faisceaux ligneux surnuméraires (s).

A chaque faisceau ligneux majeur correspondent deux faisceaux libériens (l).

$B-B^{\prime}$ : Les 6 faisceaux ligneux majeurs ( $f 1$ à fo) s'élargissent et détachent leurs ailes ( $f 1, f 2$ et fo) ou seulement une aile ( $f 4$ et $f 5$ ) ou pas du tout ( $f 3)$. Il faut noter qu'à ce stade, seule la partie centrale des faisceaux ligneux majeurs comporte un pole. 
Soulignons enfin que, même stimulées dans leur croissance, les racines latérales conservent des caractéristiques anatomiques plus proches de celles des racines latérales ordinaires que des pivots.

\title{
4. Conclusion
}

L'appareil radical des jeunes plants, tel qu'il vient d'être présenté, est un cas idéal correspondant à un ensemble qui évolue dans un environnement relativement favorable. Il n'en est pas de même dans la nature où le substrat est souvent hétérogène : présence de cailloux et différence de consistance des sols se sur-ajoutent à la présence de certains éléments de la faune souterraine et des micro-organismes pour constituer une menace permanente à ce fonctionnement hiérarchisé.

Lorsque la racine principale est déviée ou lésée, voire sectionnée, il se met en place un système de remplacement qui assure la continuité de l'ancrage et de l'absorption de l'eau et des sels minéraux par la plante. Nous verrons dans d'autres articles les modalités des réactions qui correspondent à la manifestation des différentes stratégies adaptatives de cet appareil radical que l'on pourra comparer à la situation de référence décrite ici.

Reçu le 3 avril 1987.

Accepté le 29 septembre 1987.

\begin{abstract}
Summary
Root system study in holly oak (Quercus ilex L.) seedlings whose primary root develops without amputating or diverting
\end{abstract}

The experimental study of the root system of woody plants is far behind that of the aerial system. To make up for this difference, a study was begun on a Mediterranean sclerophyllous oak

Les faisceaux libériens commencent à fusionner deux à deux (1).

$C-C^{\prime}$ : Niveau à 7 faisceaux ligneux alternant avec 7 faisceaux libériens (6 faisceaux majeurs et un supplémentaire non discernable).

$D-D^{\prime}$ : Niveau à 6 faisceaux ligneux alternant avec 6 faisceaux libériens. Les coupes $B, C$ et $D$ appartiennent au même pivot. On retrouve en $D$ les 6 faisceaux ligneux majeurs de $B$.

Tap root cross-sections at a distance of 5 (A), 7 (B), 20 (C) and 55 (D) centimeters from the cotyledons and corresponding interpretation diagrams $\left(A^{\prime}\right.$ to $\left.D^{\prime}\right)$.

$A-A^{\prime}: 4$ major xylem fascicles ( $f 1$ to $f 4$ ) can be seen between which are positionned supernumarary xylem fascicles. To each major xylem fascicle corresponds two phloem fascicles.

$B-B^{\prime}: 6$ major xylem fascicles ( $f 1$ to $\left.f 6\right)$ widen and individualize wings ( $f 1, f 2$ and $\left.f 6\right)$ or one wing only ( $f 4$ and f5) or none (f3).

At this stage only the central part of the major xylem fascicles possess a pole. Phloem fascicles are starting to unite two by two (1).

$C-C^{\prime}$ : Level where 7 xylem fascicles alternate with 7 phloem fascicles (6 major fascicles and one undistinctable additional one).

$D-D^{\prime}$ : Level where 6 xylem fascicles alternate with 6 phloem fascicles. Sections $B, C$ and $D$ belong to the same tap root. In $D$ can be seen the 6 major xylem fascicles found in $B$. 
(Quercus ilex L.). The present study defines the developmental characteristics of the root system in young seedling whose tap root has neither been diverted nor amputated and which grows in different ecological conditions (substrates, temperatures). The results obtained can be used as an interpretative guide for the root system's reactions to both diverting and amputating. These reactions will be described elsewhere. The study was made in RIEDACKER type minirhirotrons (1974) and Lamond type fog chambers (1975).

The root system of holly oak seedlings (fig. 1) consists of one main root (tap root) with positive geotropism and rapid and theoretically unlimited growth, from which thin, usually short, branch roots, either subhorizontal or oblique with slow, limited growth, spread out (fig. 5). The tap root is not rectilinear, but ondulates more or less regulary (fig. 1), both in loosely-packed substrates (peat ou loam) and in the absence of any substrate (fog chamber). The substrate's resistance to root penetration, therefore, does not cause these ondulations. The largest branch roots generally begin growth on the convex part of the ondulation (fig. 1, B and C). The tap root grows better in peat than in loam or fog chambers (fig. 2). Its growth is subject to different types of fluctuations (fig. 4). It is highly sensitive to thermic variations (fig. 2 and 3 ). A $5{ }^{\circ} \mathrm{C}$ temperature drop (from $25^{\circ} \mathrm{C}$ to $20^{\circ} \mathrm{C}$ ) decreases its growth rate by a quarter in peat and by half in loam (fig. 2). This decrease is lower in fog chambers because of their neutralizing effect.

The tap root's growth rate also fluctuates regardless of temperature, substrate (peat or loam), or lack of substrate (fig. 4). The fluctuation period varies from 2 to 7 days and is probably the expression of an irregular endogenetic rythm. Systematic ablation of branch roots as they appear has not effect on the variations in the tap root's growth rate.

Anatomy also differentiates the tap root from branch roots. The tap root usually has 6 major xylem fascicles (more rarely 4 to 8 ) directly underneath the cotyledons and connected to them in groups of three per cotyledon (fig. 6). Between these are often extra xylem fascicles which are smaller, connected to the epicotyle and disappear rapidly lower down (fig. 7, A and $\mathbf{A}^{\prime}$ ). The major xylem fascicles divide between the $3^{\text {rd }}$ and $7^{\text {th }}$ centimeter from the cotyledon by enlarging and isolating their wings (fig. 7, A and B) which can result at most in a tripling of the number of xylem fascicles. These additional fascicles vanish progressively either by fusion and/or reduction up to the $20^{\text {th }}$ centimeter from the cotyledon (fig. 7, C). Beyond this, we find the same number of xylem fascicles as at the start, i.e. only major fascicles remain (fig. $7, \mathrm{D}$ ). The number of phloem fascicles, at first twice that of the major xylem fascicles in the area of the tap root near the cotyledons (fig. 7, A and B), decreases progressively, generally by fusion, until it equals the latter beyond the $20^{\mathrm{ith}}$ centimeter from the cotyledon (fig. $7, \mathrm{D}$ ). This remarkable fluctuation in fascicle number is probably related to the period of cotyledon influence. It is not present in the branch roots, which have between 3 and 5 xylem fascicles alternating with an equal number of phlocm fascicles.

Key words : Ouercus ilex, root, development, seedling, temperature, vascular bundles.

\section{Références bibliographiques}

Aïssa D., 1981. Etude expérimentale de la germination du Chêne vert. Thèse $3^{\mathrm{e}}$ cycle, Univ. AixMarseille III.

Arbez M., 1971. Croissance des racines du pin laricio de Corse (Pinus nigra Arn. ssp laricio) au stade juvénile. Ann. Sci. For., 28, 259-288.

BARNEY C.W., 1951. Effect of soil temperature and light intensity on root growth of lobolly pine seedlings. Plant Physiol., 26, 146-163.

Boureau E., 1952. L'évolution des végétaux et l'anatomie des plantules. Ann. Biol., 28 (7-8), 163181.

Champagnat M., Baba J., Delaunay M., 1974. Corrélations entre le pivot et ses ramifications dans le système racinaire de jeunes chênes cultivés sous un brouillard nutritif. Rev. Cytol. et Biol. Vég., 37, 407-418.

COOPER A.J., 1973. Root temperature and plant growth. Research Review $\mathrm{n}^{\circ}$ 4, Commonwealth Agricultural Bureaux, $73 \mathrm{p}$.

Costes H., 1901. Flore descriptive et illustrée de la France, de la Corse et des contrées limitrophes. Librairie des Sciences Naturelles, 3 vol. 
Duchaigne A., 1951. Le passage de la racine à la tige chez les dicotylédones devant une nouvelle étude de leur ontogénie vasculaire. Thèse Doc. ès Sci., Poitiers, 134 p.

Dyanat-Nejad H., Neville P., 1973. Variation du nombre de faisceaux dans la racine principale du Cacaoyer (Theobroma cacao L.). Rev. Gén. Bot., 80, 41-74.

El Alaour H., 1978. Etude expérimentale de la morphogenèse du système racinaire du semis de Chêne-liège (Quercus suber L.). Thèse $3^{\mathrm{e}}$ cycle, Univ. Nancy I, 1-76.

Felomann L.J., 1984. Regulation of root development. Ann. Rev. Plant Physiol., 35, 223-242.

Fournier P., 1946. Les quatre flores de la France, Corse comprise. Paul Le Chevalier Ed., Paris, $1-1091$.

Gaither D.H., Lutz D.H., Forrence L.E., 1975. Abscisic acid stimulates elongation of excised pea root tips. Plant Physiol, 55, 948-949.

HEAD G.C., 1965. Studies of diurnal changes in cherry root growth and nutritional movements of apple root tips by time lapse cinematography. Ann. Bot., N.S., 29, 219-224.

Hitton R.J., Khatamian, 1974. In situ observations of woody plant root growth. Internationales Symposium Postdam, septembre 1971. Ecology and Physiology of root growth. Akademie Verlag Berlin, 171-186.

HoffmanN G., 1968. Beeinflussung des Wurzel und Sprosswachstums der Robinia (Robinia pseudoaccacia L.) durch Bodenbeheizung. Arch. Dorstives, 17, 431-453.

Hoffmann G., 1974. Einfluss von Sommerdürre auf das Wurzel und Sprosswachstum von Lärche (Larix leptolepis). Symposium Postdam, 1971. Ecology and Physiology of root growth. Akademie Verlag Berlin, 213-282.

Jackson M.B. (Editeur), 1986. New root formation in plants and cuttings. Dev. Plant Soil Sci., N.L.D., 20, 1-282.

KaZARJan V.O., Khurshudjan, 1965. The seasonal and diurnal rythm of stem and root growth of one year seedlings of Quercus robur and Eleagnus angustifoli. Fiziol. Rast., 13, 725-728 (traduction anglaise en 1966).

Kramer P.J., Koslowski T.T., 1960. Physiology of trees. Mc Graw-Hill, New York, 1-642.

LAMOND M., 1975. Dispositif de culture de plantes entières en caissons, sous brouillard nutritif en usage à Clermont-Ferrand. C. R. des Séminaires du Groupes d'Etude des Racines (19741975), Nancy-Grenoble, 2, 6-33.

Lescot M., 1967. Sur le mode de réduction du nombre de convergents dans la plantule de Cucumis melo L. Adansonia ser., 2,7 (3), 399-420.

Levy G., 1968. Importance des propriétés du sol pour l'enracinement de Picea excelsa et de Pinus silvestris. Ann. Sci. For., 25, 157-188.

Lyr H., Hoffmann G., 1967. Growth rate and growth periodicity of trees roots. Int. Rev. For. Res., 2, 181-236.

Priestley J.H., Evershed A.F.C.H., 1922. Growth studies. I. A quantitative study of the growth of roots. Ann. Bot., 36, 225-237.

Priestley J.H., Pearsall W.H., 1922. Growth studies. II. An interpretation of some growth curves. Ann. Bot., 36, 239-249.

RIEDACKer A., 1974. Un nouvel outil pour l'étude des racines et de la rhizosphère : le minirhizotron. Ann. Sci. For., 31, 129-134.

Riedacker A., 1976a. Etude des possibilités de modifier la morphogenèse juvénile des systèmes racinaires de quelques espèces forestic̀res; applications pratiques. Proc. XVI IUFRO World Congres, Oslo, juin 1976, Div. I, 376-387.

Riedacker A., 1976b. Les déformations racinaires. Comptes Rendus des Séminaires du Groupe d'Etude des Racines, 4, $2^{\prime \prime}$ partie, 93-105.

Riedacker A., 1976c. Rythmes de croissance et de régénération des racines des végétaux ligneux (Etude bibliographique). Ann. Sci. For., 33, 103-138.

Riedacker A., 1978. Etude de la déviation des racines horizontales ou obliques issues de boutures de peuplier qui rencontrent une obstacle : application pour la conception de conteneurs. Ann. Sci. For., 35, 1-18.

Schwartz O., 1964. Quercis, in Tutin T.G. (ed.), Flora Europea, 1, 61-64. 
SeELiger, 1956. Über die Kultur isolierter Wurzeln der Robinie (Robinia pseudoaccacia L.). Flora, 144, 47-83.

Souid C., Gagnaire-Michard J., 1974-1975. Enracinement de la bouture de Peuplier (Populus nigra). Morphogenèse - Absorption racinaire et foliaire des éléments minéraux. Premiers résultats. C. R. des Séminaires du Groupe d'Etude des Racines, 2, 191-199.

TAVAKOL R., 1979. Etude de la morphogenèse de systèmes racinaires issus de semis et boutures de Chênes et de Peupliers. Applications pratiques pour la production de plants. Thèse. Université de Nancy I, $127 \mathrm{p}$.

TAYLOR H.M., HuCK M.G., KLIPPER B., LUND Z.F., 1970. Measurements of soil grown roots in a rhizotron. Agronomy Journal, 62, 807-809.

Tierz A., 1975. Einfluss der Kulturmethode auf die Ausscheidung von Phytohormonen durch Erbsenwurzeln. Biochem. Physiol. Pflanzen, 167, 371-378.

TolneN F.G., 1977. Morphogenèse des systèmes racinaires juvéniles du Pin Laricio et du Cèdre. Mémoire de $3^{z}$ année de l'Ecole Nationale des Ingénieurs des Travaux des Eaux et Forêts, I.N.R.A. Nancy, Doc. 77, 3, 1-81.

TORRey J.G., 1965. Physiological bases of organization and development in the root. Encyclop. Plant Physiol., 15, 1256-1327.

Troncher A., 1930. Recherche sur les types d'organisation les plus répandus de la plantule des dicotylédones, leurs principales modifications, leurs rapports. Archives Botaniques, Tome $I V$, mémoire $n^{\circ} 1,1-252$. 